\title{
Un nouveau dispositif pour la caractérisation du retrait et du gonflement des sols argileux
}

\author{
Jean-Bernard Kazmierczak ${ }^{1, \star}$, Tatiana Maison ${ }^{1,2,3}$, Farid Laouafa $^{1}$, Patrice Delalain ${ }^{1}$ et \\ Jean-Marie Fleureau ${ }^{3}$ \\ ${ }^{1}$ INERIS, Parc Technologique Alata, 60550 Verneuil-en-Halatte, France \\ 2 B2R, Institut Polytechnique LaSalle Beauvais, Département Géosciences, 19, rue Pierre-Waguet, 60000 Beauvais, France \\ 3 CentraleSupélec, Laboratoire MSSMat, CNRS UMR 8579, Grande Voie des Vignes, 92295 Châtenay-Malabry cedex, France
}

\begin{abstract}
Résumé - Les phénomènes de retrait et de gonflement des argiles induisent chaque année des dégâts sur les structures d'habitation. Leur compréhension et leur quantification constituent donc deux objectifs importants pour réduire la sinistralité. C'est dans ce contexte qu'a été développé, au sein d'un microscope électronique à balayage environnemental (MEBE), un nouveau dispositif pour caractériser le retrait et le gonflement de matériaux argileux. Il permet d'établir la relation qui lie la variation de volume d'un micro-échantillon à sa variation de teneur en eau, très rapidement. Associé à un modèle analytique qui s'appuie sur des profils types de pénétration de la sécheresse dans le sol, ce nouvel essai permet d'estimer l'amplitude du tassement et/ou du gonflement attendu à l'échelle d'un ouvrage.
\end{abstract}

Mots clés : caractérisation des argiles / retrait / gonflement / dispositif expérimental

\begin{abstract}
A new device to analyse the shrinkage and swelling of clayey soils. Each year, the shrinkage and swelling of clays induce damages on housing structures. Their understanding and their quantifying constitute two important objectives to reduce damages. It is in this context that was developed a new device to characterise the swelling-shrinkage behaviour of these clayey materials within an Environmental Scanning Electron Microscope (ESEM). It allows to construct a relationship between the volume variation of a microsample and its water content variation in a very short time. Associated with an analytical model based on standard profiles of drought penetration in the soil, this new test allows to estimate the amplitude of settlement and/or swelling at the house scale.
\end{abstract}

Keywords: clayey soil characterisation / shrinkage / swelling / experimental device

\section{Introduction}

Sous l'effet des conditions climatiques saisonnières, les sols argileux peuvent présenter des déformations volumiques importantes, en fonction de la modification de leur teneur en eau. Celles-ci ont parfois de graves conséquences sur les constructions en surface, notamment quand elles provoquent des déformations différentielles.

En France, le phénomène de retrait-gonflement a conduit à 4,3 G€ d'indemnisation sur les 15 dernières années dans le cadre du régime des catastrophes naturelles. Plus de 8000 communes françaises ont été touchées pour le seul été 2003 (Vincent et al., 2008, 2009).

Les phénomènes physiques à l'origine de ces problèmes sont intrinsèquement complexes et difficiles à reproduire numériquement compte tenu de l'échelle spatiale à laquelle

\footnotetext{
* Auteur correspondant :

jean-bernard.kazmierczak@ineris.fr
}

ils se produisent (absorption de l'eau entre les feuillets microscopiques d'argile et de la physique mise en jeu). Par ailleurs, le comportement de ces sols est également affecté par des facteurs extérieurs liés par exemple à la présence de végétation et/ou des ouvrages (maisons individuelles) eux-mêmes qui modifient l'état de contrainte dans les sols concernés.

On peut toutefois évaluer, dans certaines configurations, le tassement et le gonflement d'un massif de sol dus au changement de sa teneur en eau grâce, notamment, à un des modèles élastiques (Fredlund et Rahardjo, 1993) ou élastoplastiques (Alonso et al., 1990 ; Cui et Delage, 1996). Ceux-ci permettent, dans un cadre couplé, de déterminer les déformations engendrées à la fois par la contrainte effective et l'effet de la succion.

L'utilisation de ces outils numériques nécessite la détermination de nombreux paramètres qui s'appuient en général sur des essais de laboratoire réalisés sur des échantillons de sols argileux dont le volume avoisine une dizaine de centimètres cubes. 
Compte tenu de leurs dimensions et de la cinétique très lente du phénomène, les effets du retrait et du gonflement sur de tels échantillons peuvent nécessiter des temps de réaction et d'équilibre relativement longs, de l'ordre de la dizaine de jours (Plat et al., 2009).

Pour pallier cet inconvénient, une méthode de caractérisation du retrait et du gonflement d'un matériau argileux au sein d'un microscope électronique à balayage environnemental (MEBE) est proposée. Le MEBE est un microscope dans lequel il est possible de maîtriser l'humidité relative HR et d'observer en temps réel, entre autres, l'évolution de la surface d'un microéchantillon de sol de très petit volume (quelques micromètres cubes). Celui-ci est donc très réactif, réactivité liée à la dimension de l'échantillon.

L'observation de la variation de volume doit obligatoirement être associée à celle de la variation de poids de l'échantillon pour qu'une relation entre ces deux variables puisse être établie. Dans cet objectif, un dispositif de micropesage de l'échantillon à l'intérieur du MEBE a été développé.

La variation de poids de l'échantillon étant dans ce contexte uniquement liée à sa variation de teneur en eau, il devient alors possible de définir la courbe reliant la variation de volume du sol à sa variation de teneur en eau, sur la base d'un essai de très courte durée, de l'ordre de la journée.

Un modèle analytique simplifié a pu être formulé sur la base de ces mesures et utilisé sous certaines hypothèses pour relier le comportement (de retrait-gonflement) de l'échantillon microscopique à celui du massif de sol de manière à évaluer la réponse en grand du massif à des modifications de sa teneur en eau.

\section{Démarche générale}

Parmi les paramètres nécessaires à la mise en œuvre de cette approche, la relation, reliant la variation de volume (ou de surface) du matériau à sa teneur en eau, constitue l'information clé.

Cette relation peut être définie à partir d'un essai au MEBE qui est réalisé sur une très petite quantité de matériau (quelques milligrammes). La question de la représentativité de l'échantillon se trouve ici amplifiée par comparaison aux échantillons plus «volumineux » de laboratoire mais il a été montré par Maison et al. (2009a,b) qu'il n'y avait pas d'effet d'échelle. Par ailleurs, la vitesse d'exécution de l'essai qui en découle constitue un avantage majeur permettant de multiplier les tests.

De nombreux essais «classiques » de laboratoire permettent déjà d'estimer la potentialité de retrait et de gonflement des matériaux argileux. Les plus intéressants sont rappelés par Plat et al. (2009) dans le cadre du programme ANR-ARGIC. On peut citer parmi ceux-ci :

- l'essai de chargement-déchargement sous succion contrôlée ;

- l'essai d'humidification-séchage sous chargement mécanique contrôlé ;

- les essais de dessiccation sur échantillons intacts : limite de retrait et retrait linéaire ;

- la mesure de potentiel de gonflement ou de retrait ;

- l'essai de retrait, suivi par pesée (Makki et al., 2008).
La plupart de ces essais, notamment ceux qui sont réalisés sous succion contrôlée, (c'est-à-dire, en ayant une connaissance précise de la teneur en eau au sein du matériau), peuvent durer jusqu'à deux mois, compte tenu des temps d'équilibre associés au volume des échantillons. Ce type d'essai présente également un coût non négligeable (plus de 500 euros l'essai).

Dans le MEBE, la quantité d'argile testée est très faible et l'équilibre hydrique au sein de l'échantillon est atteint très rapidement (en une heure maximum, selon l'argile ou le sol argileux testé) pour chaque palier hydrique. La courbe peut ainsi être rapidement construite. Le problème qui doit toutefois être résolu concerne la formulation de la relation entre l'humidité relative HR qui règne au sein de la chambre du microscope et la teneur en eau $\mathrm{w} \%$ dans le matériau. Pour ce faire, nous avons mis au point un dispositif de pesée au sein du MEBE (Fig. 1). Il a fait l'objet d'un dépôt de brevet $n^{\circ}$ FR1054771 (Kazmierczak et al., 2010).

Celui-ci est constitué de quatre éléments principaux :

- un dispositif permettant d'imposer une température donnée à l'échantillon. Il s'agit d'un module à effet Peltier, solidaire d'un échangeur thermique. Ce dernier transmet vers l'extérieur du MEBE les calories extraites de l'échantillon pour le maintenir à une température donnée ;

- un capteur de force qui permet une mesure jusqu'à $2 \mathrm{~N}$, avec une résolution de $0,01 \mathrm{~N}$;

- un bras de levier permettant d'amplifier la variation de poids de l'échantillon, grâce à l'excentrement de la charge ;

- un dispositif d'acquisition permettant d'enregistrer les variations de poids de l'échantillon, avec une résolution de $0,5 \mathrm{mg}$.

\subsection{Principe de l'essai}

L'essai consiste à faire varier au sein du MEBE, à température constante $\mathrm{T}$, la pression $\mathrm{P}$, de manière à imposer un état hygrométrique connu HR. À partir du diagramme de phases de l'eau (Fig. 2), on impose ainsi à l'échantillon des humidités relatives $H R(T, P)$ variant entre 5 et $95 \%$.

Pour chaque état hydrique, on observe la variation de la déformation surfacique $(\Delta S)$ et de poids de l'échantillon. Cette variation est obtenue par le traitement informatique du différentiel des images de la taille des agrégats de sol argileux enregistrées grâce au MEBE. La variation volumique de l'échantillon sera ensuite calculée sur la base d'hypothèses décrites à la Section 4.3.

Sur la base de la combinaison des courbes (obtenues indépendamment) décrivant $\Delta S=\mathrm{f}(H R)$ et $w=\mathrm{f}(H R)$, il devient alors possible de construire la courbe de variation de la déformation surfacique de l'échantillon $\Delta S(\%)$ en fonction de la teneur en eau $w(\%)$.

\subsection{Protocole d'essai}

Le déroulement de l'essai est décrit ci-après. Il consiste en : - un étalonnage du système de pesage sur la base de masses étalon connues ;

- le pesage initial de l'échantillon au sein du MEBE (ouvert) dans les conditions ambiantes (humidité relative $H R_{0}$, température $T_{0}$ et pression $P_{0}$ de la pièce) ; 


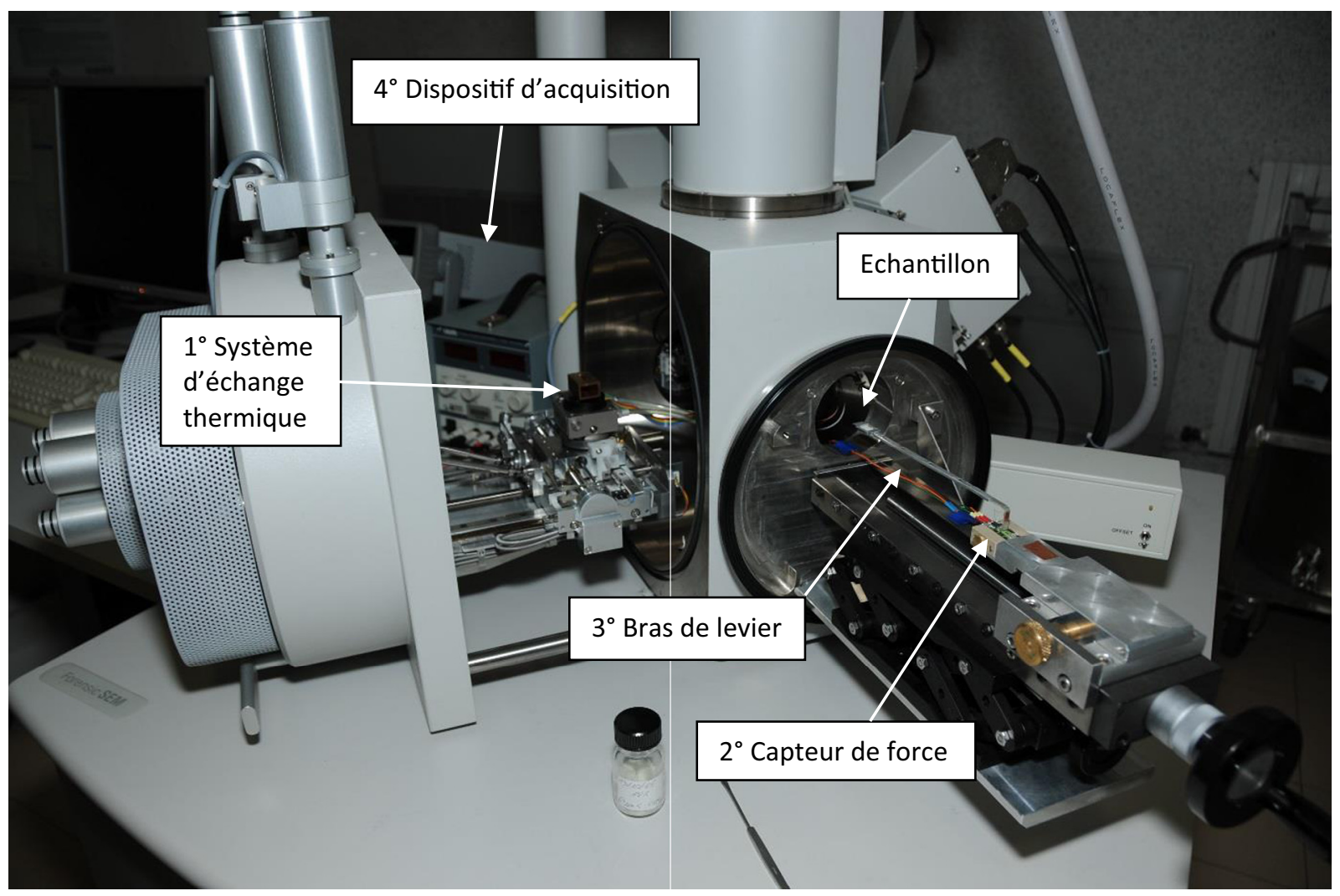

Fig. 1. Dispositif de pesée de l'échantillon développé dans le microscope électronique à balayage environnemental (MEBE).

Fig. 1. Weighing device of the sample developed in the Environmental Scanning Electron Microscope (ESEM).

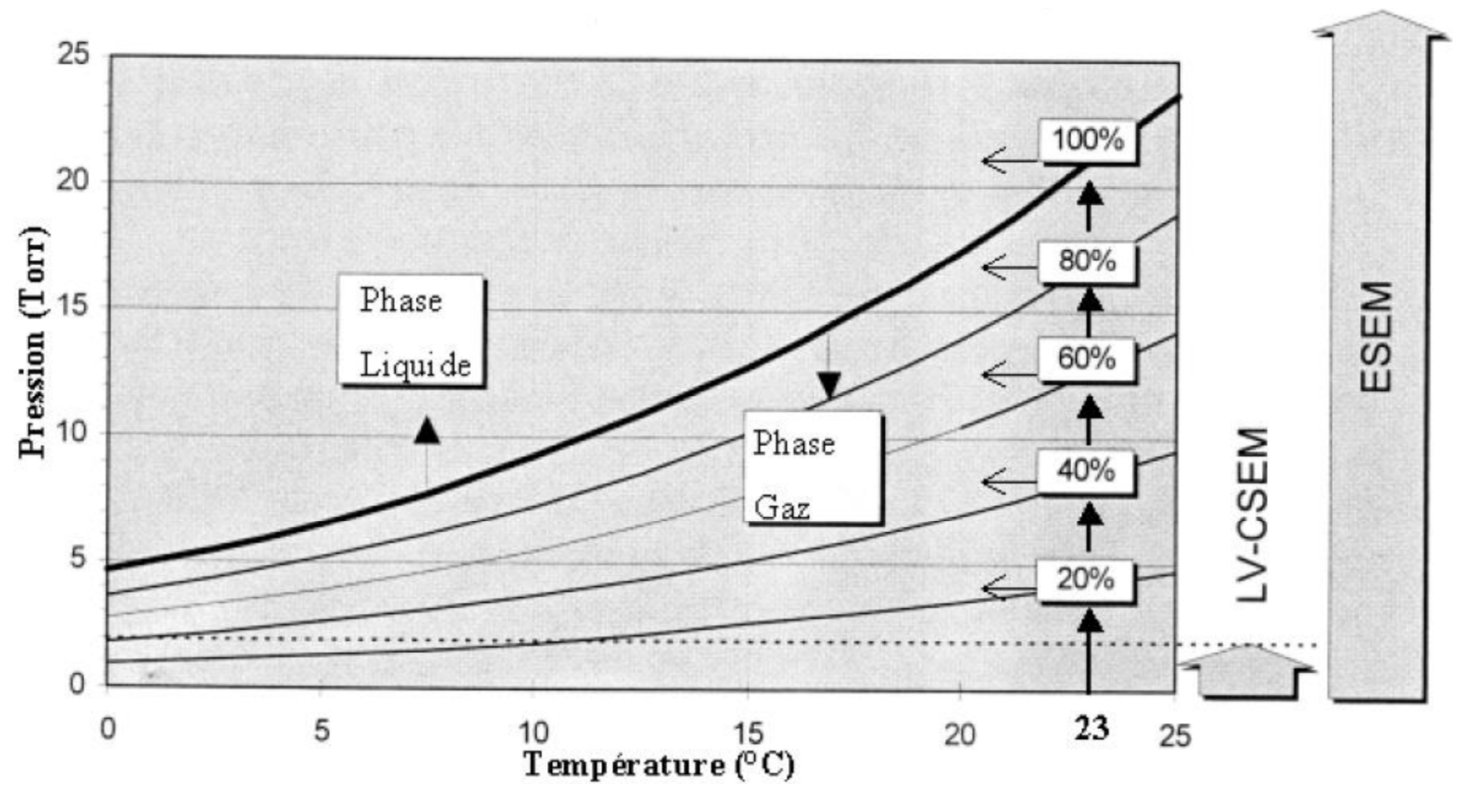

Fig. 2. Diagramme de phases de l'eau.

Fig. 2. Water phases diagram (Montes, 2002).

- la fermeture de la chambre du MEBE;

- la remise en conditions «ambiantes » de l'échantillon (température et pression dans le MEBE reconstituant l'humidité relative initiale $\mathrm{HR}_{0}$ du laboratoire) ;
- l'application successive de paliers (Fig. 3) d'humidité relative connus (séchage : $H R$ variant de $H R_{0}$ à $5 \%$ puis humidification : $H R$ variant de $5 \%$ à environ $95 \%$, puis retour à $H R_{0}$ ) avec pesage ; 


\section{Application des paliers d'humidité relative}

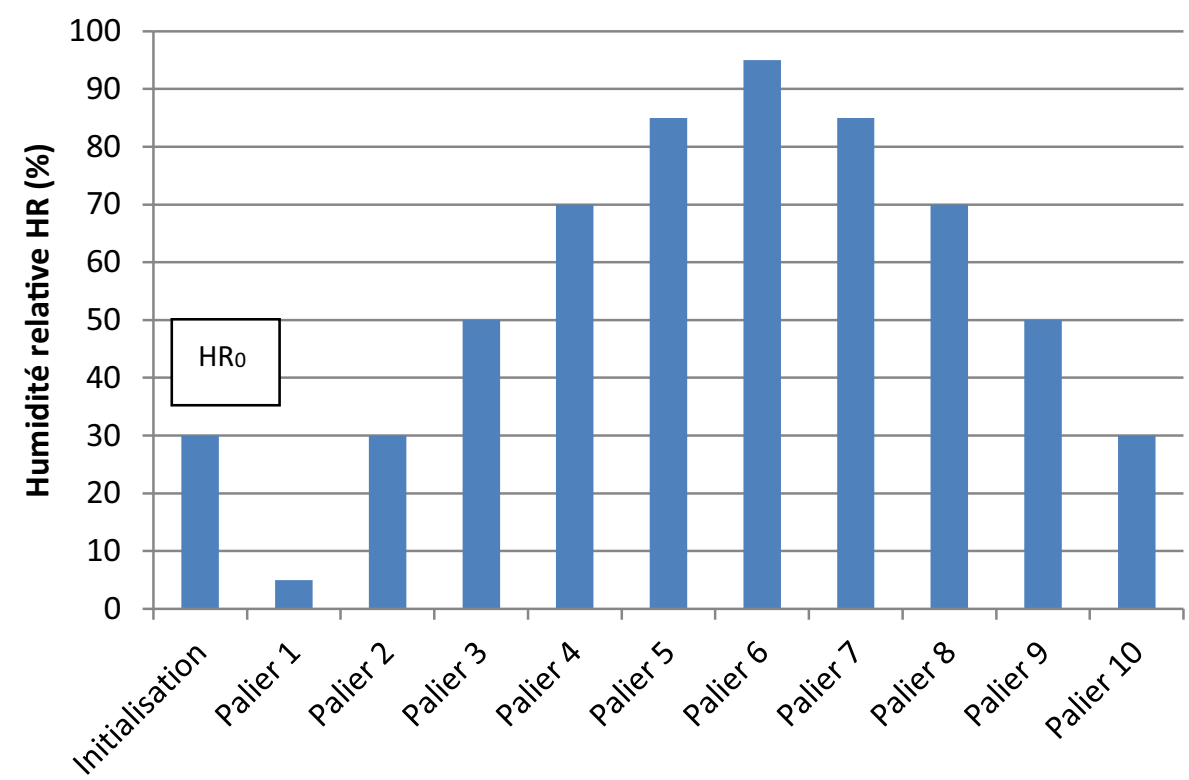

Fig. 3. Illustration des paliers d'humidité relative appliqués durant l'essai.

Fig. 3. Illustration of the relative humidity steps used in the test.

- chaque palier est maintenu jusqu'à stabilisation du poids de l'échantillon (variation inférieure à $\pm 1 \mathrm{mg}-60$ minutes environ selon la valeur de $H R$ et le matériau testé).

Pour chaque palier d'humidité $H R_{\mathrm{i}}$, des images sont enregistrées et un pesage de l'échantillon est réalisé simultanément dans le MEBE. Une analyse d'images successives permet ensuite de déterminer la variation de surface de l'échantillon (Maison et al., 2008, 2009a, b) et de l'associer à la variation de son poids. La teneur en eau $w_{0} \%$ de l'échantillon dans les conditions initiales étant connue, il est alors possible de tracer la courbe de variation de surface en fonction de la teneur en eau $\Delta S=\mathrm{f}(w \%)$.

\subsection{Avantages et inconvénients de la méthode}

Ce système présente l'avantage majeur de permettre la réalisation d'un cycle complet dans un temps très court (de l'ordre de 24 h). Á partir d'un modèle analytique (Sect. 4), il devient possible d'estimer rapidement l'amplitude des déformations que pourrait avoir à supporter une structure construite au droit de la zone de prélèvement.

La méthode présente toutefois quelques inconvénients :

- l'essai est innovant et sa validité doit être éprouvée sur différents types de sols argileux prélevés sur des sites connus et instrumentés ;

- les résultats sont dépendants de la faible quantité de matière utilisée, ce qui peut entraîner une certaine variabilité même si la reproductivité d'un essai sur un même échantillon est très élevée ;

- le MEBE est un appareil de haute technologie qui nécessite une grande compétence de l'opérateur ;

- le coût de l'essai est donc pour l'instant élevé ; de l'ordre de 1000 euros compte tenu de l'amortissement d'un matériel onéreux et d'une main-d'œuvre qualifiée ;
- le dispositif de pesée, très précis, est fragile, à l'instar du MEBE.

\section{Exemple d'application sur la Montmorillonite grecque}

Plusieurs essais ont été réalisés en suivant le protocole décrit précédemment. Nous présentons dans ce qui suit des résultats obtenus avec la Montmorillonite grecque pour laquelle de nombreux essais de caractérisation de laboratoire existent (Fleureau et al., 1992).

La Figure 5 illustre la variation de la teneur en eau dans l'échantillon de Montmorillonite grecque en fonction de l'humidité relative imposée dans le MEBE. Le pas de mesure de la teneur en eau est de 15 secondes. On constate qu'un temps de stabilisation de $1 \mathrm{~h}$ par palier est nécessaire. Toutefois, une forte proportion de la variation de teneur en eau intervient très rapidement à chaque palier. Pour des faibles humidités relatives, plus de $60 \%$ de la variation totale de poids est atteinte après seulement dix minutes.

Ces résultats peuvent être comparés (Fig. 4) à ceux proposés par Fleureau et al. (1992), qui les a obtenus par la méthode des plaques tensiométriques et des membranes osmotiques pour $H R>98 \%$ et par des dessiccateurs classiques pour $H R<98 \%$. Nos résultats ne concernent qu'un domaine de succion restreint $\left(p F^{\mathrm{a}}>4,8\right)$ compte tenu de la difficulté pour le MEBE d'atteindre des humidités relatives supérieures à $95 \%$.

La Figure 4 montre que les résultats fournis par le nouveau type d'essai sont en bon accord avec ceux de la littérature dans la gamme d'humidités relatives qui nous

${ }^{\mathrm{a}} p F=1+\log$ (succion, $\left.\mathrm{kPa}\right)$. 


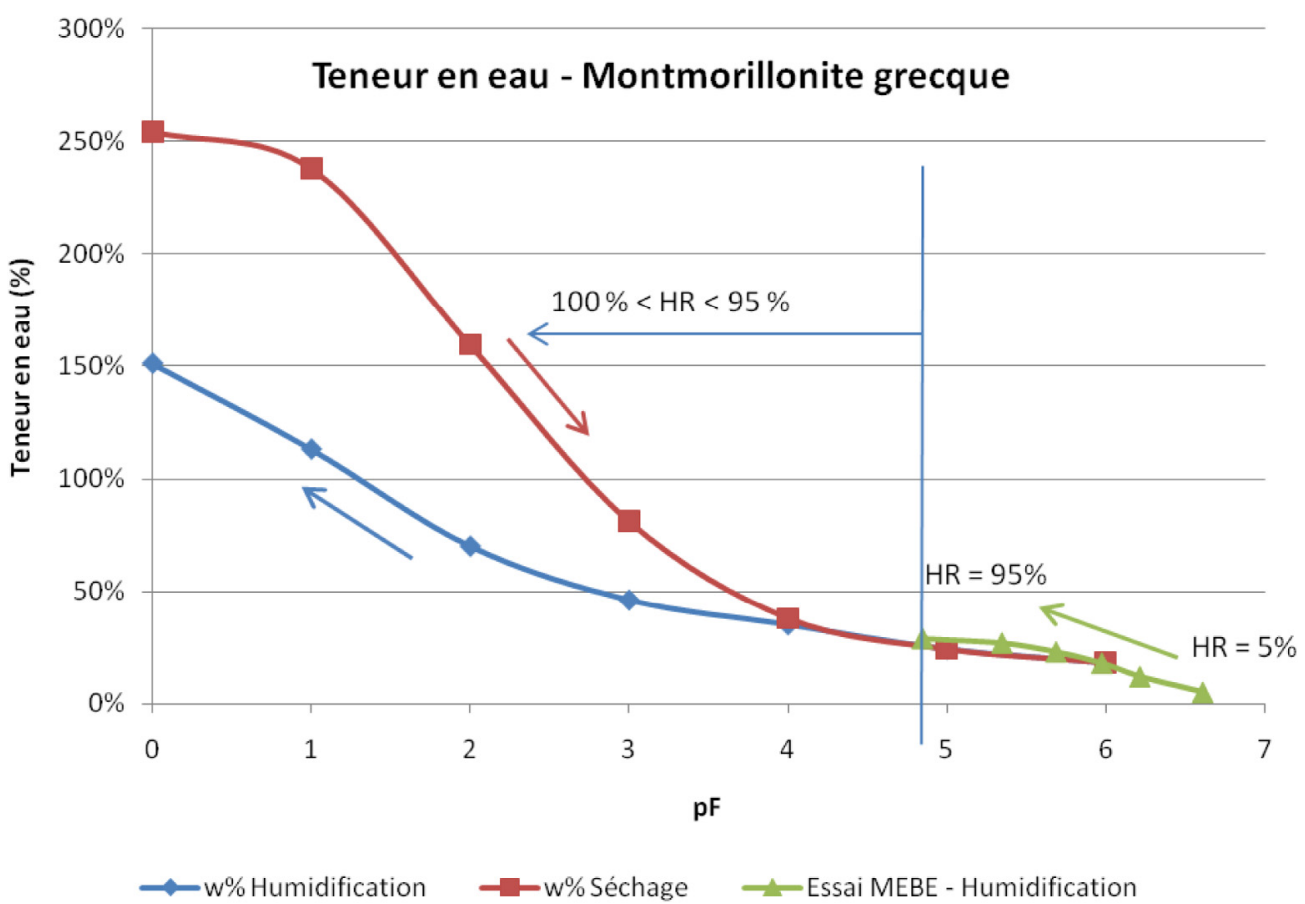

Fig. 4. Variation de la teneur en eau en fonction de la succion.

Fig. 4. Change in water content as a function of suction.

D'après Fleureau et al. (1992).

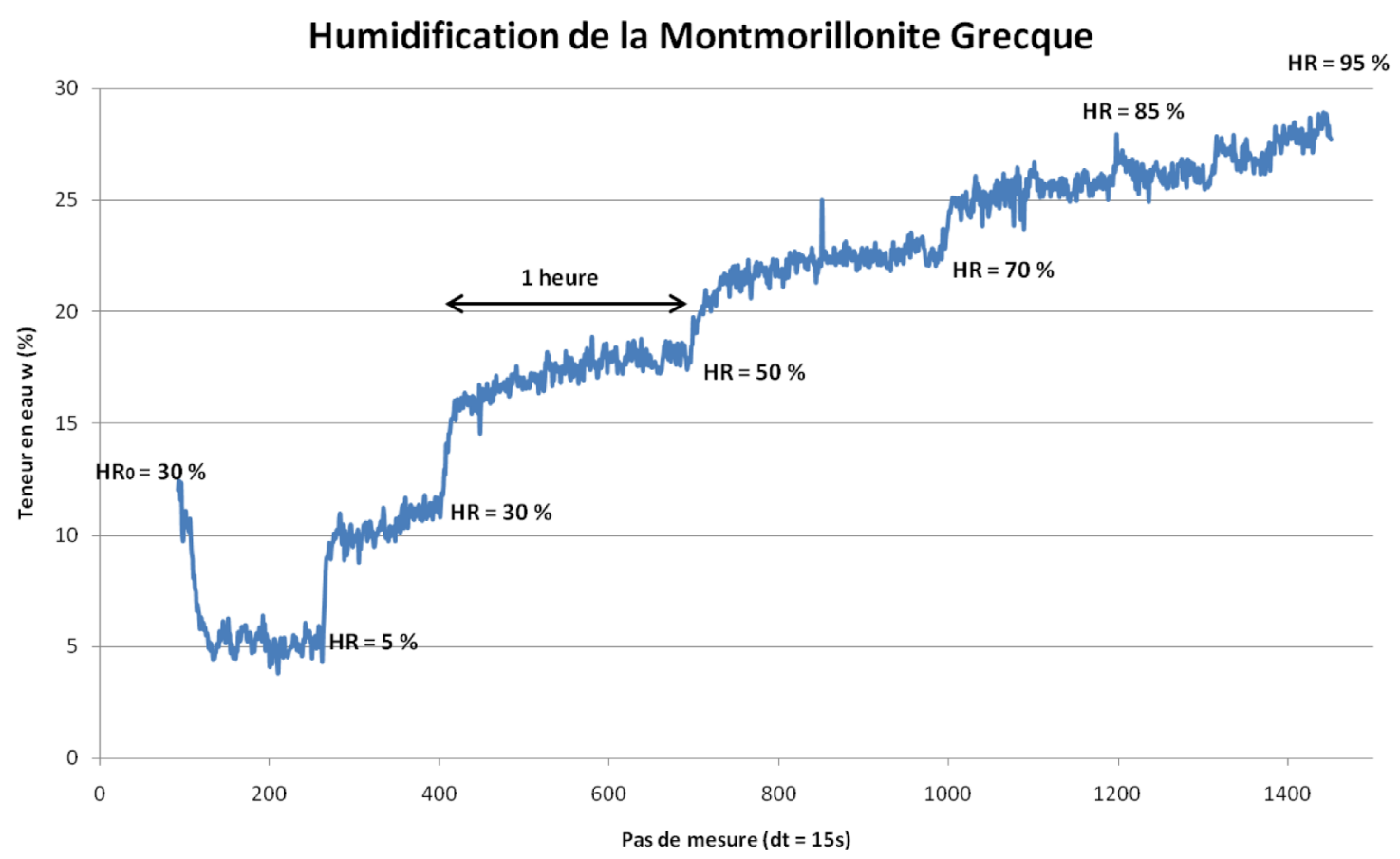

Fig. 5. Variation de la teneur en eau sous l'effet de l'humidification dans le microscope électronique à balayage environnemental (MEBE) pour la Montmorillonite grecque.

Fig. 5. Evolution of water content induced by wetting in the Environmental Scanning Electron Microscope (ESEM), for Greek Montmorillonite.

intéresse (de 5 à $95 \%$ ), bien qu'ils soient réalisés, respectivement à des échelles micro et méso.

La démarche utilisée pour obtenir une représentation de la fonction $\Delta S=\mathrm{f}(w \%)$ (Fig. 8) est la suivante. Il s'agit tout d'abord de construire la courbe de variation de la teneur en eau $w \%$ en fonction de $H R$ (Fig. 6) à partir des mesures de poids de l'échantillon effectuées grâce au nouveau dispositif de pesée. Ces mesures sont illustrées sur la Figure 5. Il s'agit ici de déterminer à partir de l'évolution du poids de l'échantillon, l'évolution de la teneur en eau et de représenter celle-ci pour chaque valeur de HR 


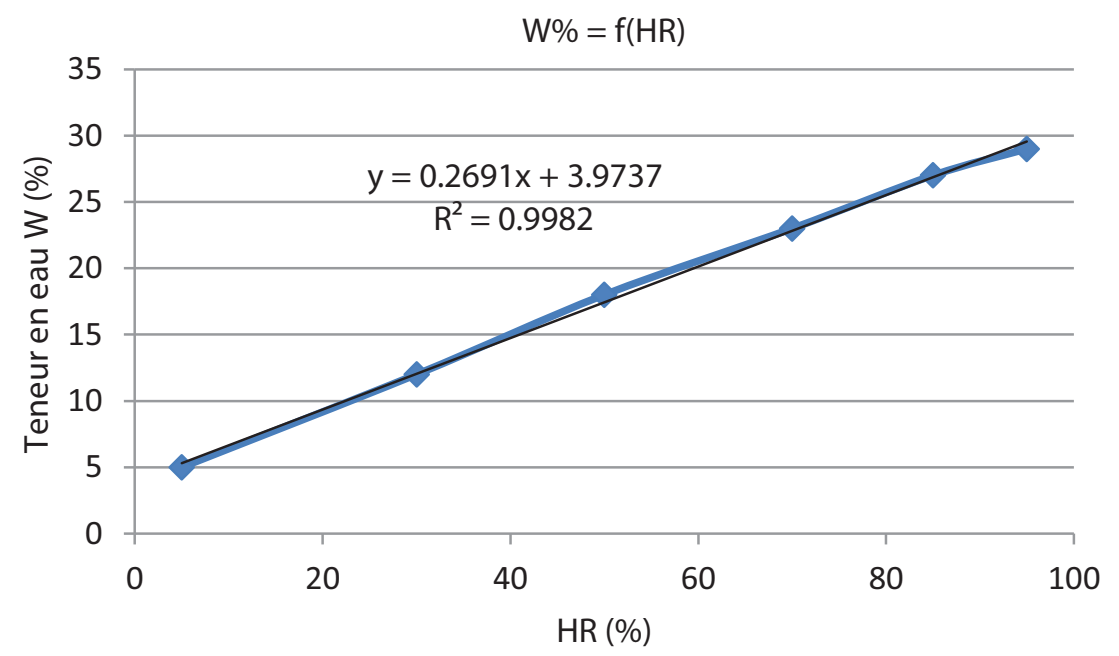

Fig. 6. Évolution de la teneur en eau de la Montmorillonite grecque en fonction de l'humidité relative $H R$ dans le microscope électronique à balayage environnemental (MEBE).

Fig. 6. Evolution of the water content for Greek Montmorillonite as a function of relative humidity RH.

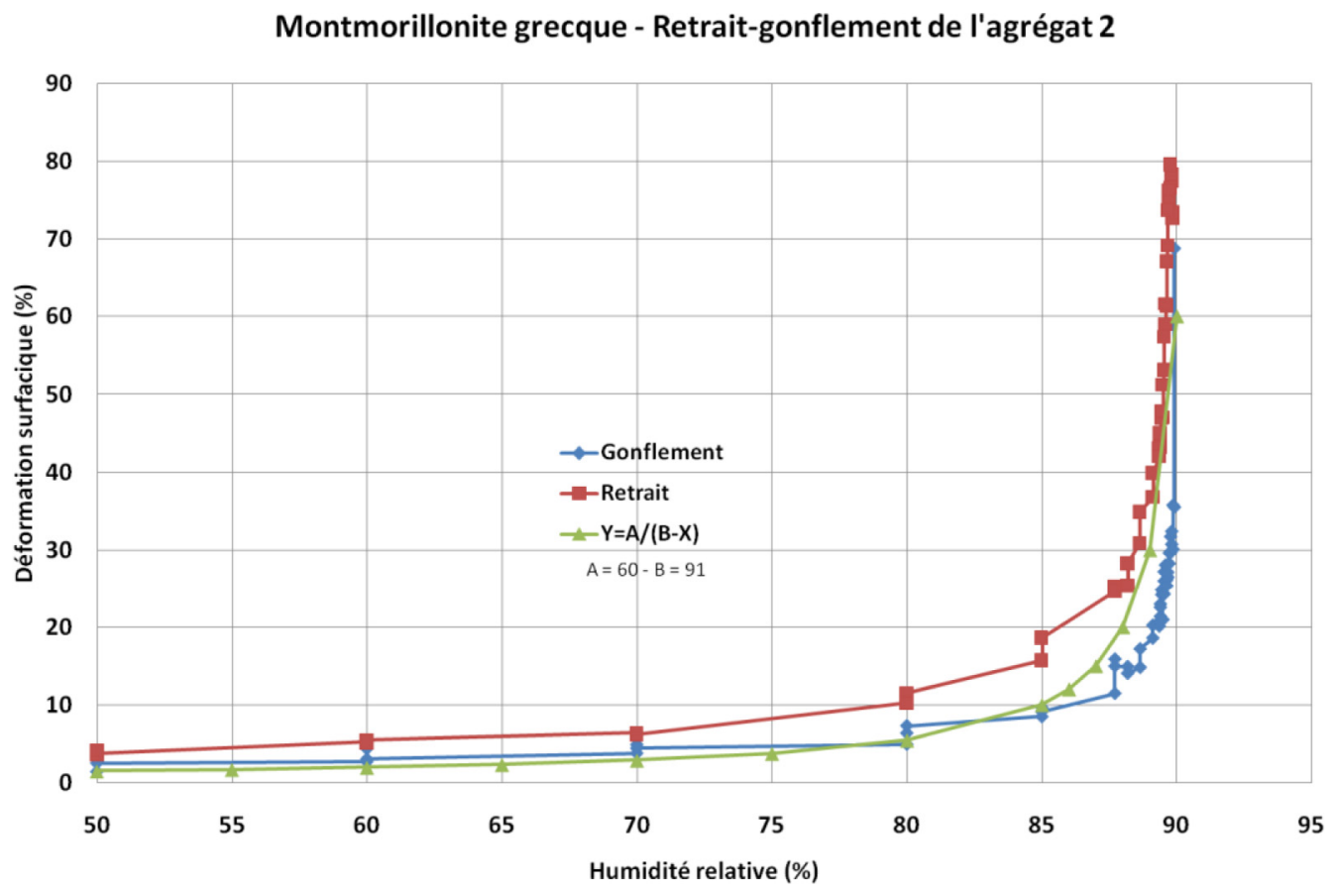

Fig. 7. Variation de surface de la Montmorillonite grecque en fonction de l'humidité relative $H R$.

Fig. 7. Surface variation of the Greek Montmorillonite as a function of the relative humidity RH.

imposée dans l'essai (Fig. 6). Comme première approximation, nous avons proposé une relation de la forme $w=A_{1} \times H R+B_{1}$ qui permet d'approximer le résultat expérimental (ici $A_{1}=0,2691$ et $B_{1}=3,9737$, obtenu après calage).

Il convient ensuite d'analyser les images des agrégats argileux enregistrées grâce au MEBE pour chaque valeur de $H R$. Le différentiel de surface des agrégats observés permet de calculer et de représenter la déformation surfacique du matériau en fonction de l'humidité relative $H R$ (Fig. 7). Une équation de la forme $\Delta S=A_{2} /\left(B_{2}-H R\right)$ permet d'approximer les résultats expérimentaux obtenus (ici $A_{2}=60$ et $B_{2}=91$ ).
La combinaison des deux résultats expérimentaux permet de décrire la relation $\Delta S=\mathrm{f}(w \%)$ sous la forme suivante :

$$
\Delta S=\frac{A_{1} \times A_{2}}{A_{1} \times B_{2}+B_{1}-w}
$$

et de tracer la courbe de la Figure 8.

On constate (pour cet exemple) qu'une augmentation de dix points (entre 15 et $25 \%$ ) de teneur en eau conduit à une augmentation de la surface de l'échantillon de l'ordre de $3 \%$, 


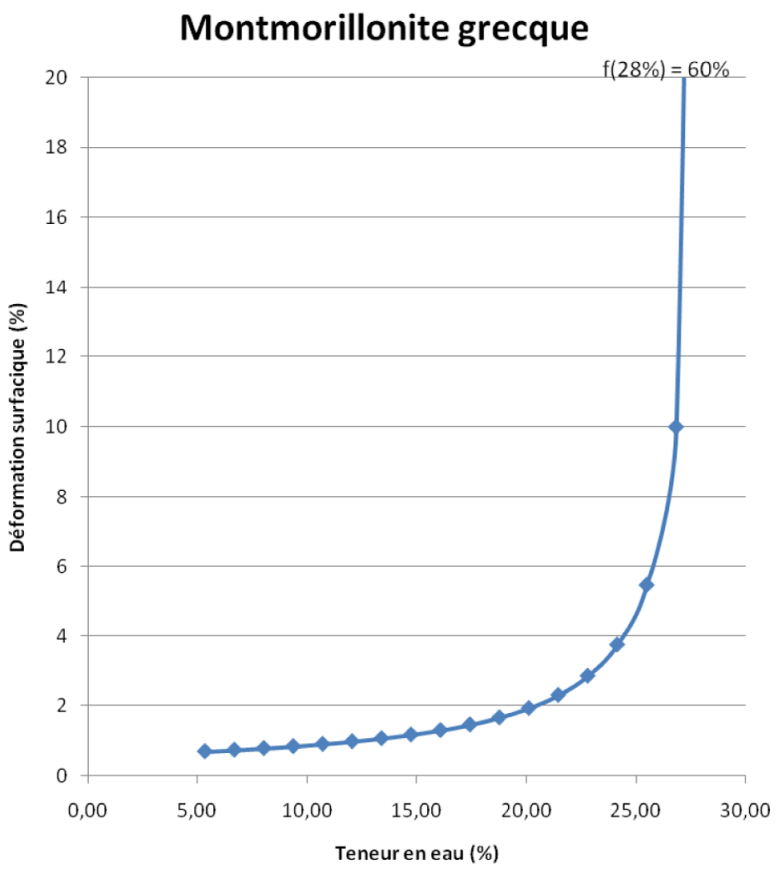

Fig. 8. Courbe reliant la variation de la déformation surfacique à la teneur en eau pour la Montmorillonite grecque.

Fig. 8. Curve relating the change in surface strain to the water content for the Greek Montmorillonite.

et que le passage de $w$ de 25 à $28,2 \%$, conduit à une augmentation d'environ $(60-4)=54 \%$ !

\section{4 Évaluation du retrait-gonflement d'un massif - Modèle analytique}

Pour exploiter pleinement le nouveau dispositif expérimental et transcrire son apport au comportement à l'échelle d'un ouvrage, nous nous sommes attachés à construire un « modèle » permettant le calcul du tassement ou du gonflement d'un massif de sol soumis à des variations de teneur en eau.

Le principe de ce modèle est très simple. Il consiste à sommer analytiquement, sur toute l'épaisseur de la couche, les variations élémentaires de hauteur de chaque portion élémentaire de couche de sol. Ces variations élémentaires sont calculées sur la base de la variation de la teneur en eau à chaque profondeur du massif, via la courbe expérimentale $\Delta S=\mathrm{f}(\Delta w \%)$ obtenue précédemment.

Un certain nombre d'hypothèses, parfois fortes, limitent le champ d'application de ce modèle (contraintes induites par un bâtiment en surface, etc.).

\subsection{Principales hypothèses du modèle utilisé}

Le modèle analytique développé ici se base sur les différentes hypothèses suivantes :

- le mécanisme de retrait et de gonflement des sols argileux suit le même chemin de déformation et il est indépendant de l'échelle d'observation (Maison et al., 2008, 2009a,b), même si sa cinétique ne l'est pas (temps de réaction d'autant plus grand que le matériau testé est volumineux) ;
- la courbe de variation de surface (ou de volume) du matériau, en fonction de sa teneur en eau, peut être définie au sein du MEBE; le phénomène de retrait ou de gonflement est peu dépendant de l'état de contrainte, dans la gamme de profondeur considérée ${ }^{\mathrm{b}}$ (Bigot et Zerhouni, 2000);

- une relation entre la variation de volume du sol en champ libre et ses équivalents surfacique et linéique peut être formulée (Sect. 4.3) ;

- des profils types de pénétration de la sécheresse dans le sol peuvent être proposés (Sect. 4.4).

Ces différentes hypothèses, fortes pour certaines d'entreelles, et qui seront explicitées ci-après au fur et à mesure de la démarche décrite, permettent alors de calculer à chaque profondeur la variation d'épaisseur de la couche considérée en fonction des conditions hydriques. L'intégration de ces variations sur toute la hauteur de la couche renseigne ainsi sur les amplitudes de retrait ou de gonflement prévisibles en surface. Un lien entre le comportement microscopique (MEBE) et macroscopique (échelle de l'ouvrage) est ainsi proposé. Il s'appuie sur les observations faites sur plusieurs argiles dans le cadre de travaux de recherche menés par Maison et al. (2008, 2009a,b) et Maison (2011).

\section{2 Évaluer l'amplitude du tassement et du gonflement dans le sol}

Une information indispensable pour la détermination de l'amplitude du retrait ou du gonflement en surface est la profondeur de pénétration de la sécheresse, et donc la variation d'humidité en fonction de la profondeur qui en résulte dans le massif. Cela nécessite de disposer de deux données :

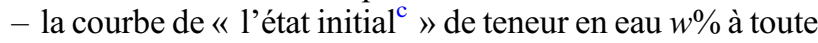
profondeur ;

- le nouvel état de $w \%$ ou la variation de cet état à toute profondeur.

À titre d'exemple, la Figure 9 illustre un cas qui s'appuie sur les mesures réalisées sur le site de Pessac dans le cadre du programme ANR-ARGIC (Vincent et al., 2009) :

- pour l'état initial (courbe $[A]$ ), les mesures montrent des valeurs de teneur en eau $(w \%)$ variant linéairement de 0 à $16 \%$ entre 0 et $0,5 \mathrm{~m}$ puis une valeur constante de $16 \%$ au-delà ;

- les états finaux après « séchage » et « humidification » sont représentés par les courbes $[B]$ de la Figure 9 ;

- les courbes $[C]$ des mêmes figures représentent la variation de teneur en eau dans le milieu entre l'état initial et les états « sec » et « humide ».

Pour ce site, on constate que la variation maximale de $27 \%$ de teneur en eau apparaît à la profondeur de 0,5 m.

La relation expérimentale, qui relie la variation de surface à la variation de teneur en eau du matériau, permet en théorie de calculer le tassement et/ou le gonflement à toutes

\footnotetext{
${ }^{\mathrm{b}}$ La pression de gonflement des argiles est en général supérieure à l'état de contrainte vertical rencontré aux faibles profondeurs.

${ }^{\mathrm{c}}$ L'état initial peut par exemple correspondre à l'état hydrique du massif durant la saison où un bâtiment a été construit. Il s'agit en fait d'un état représentatif d'une situation donnée.
} 


\section{Cycle "séchage"}

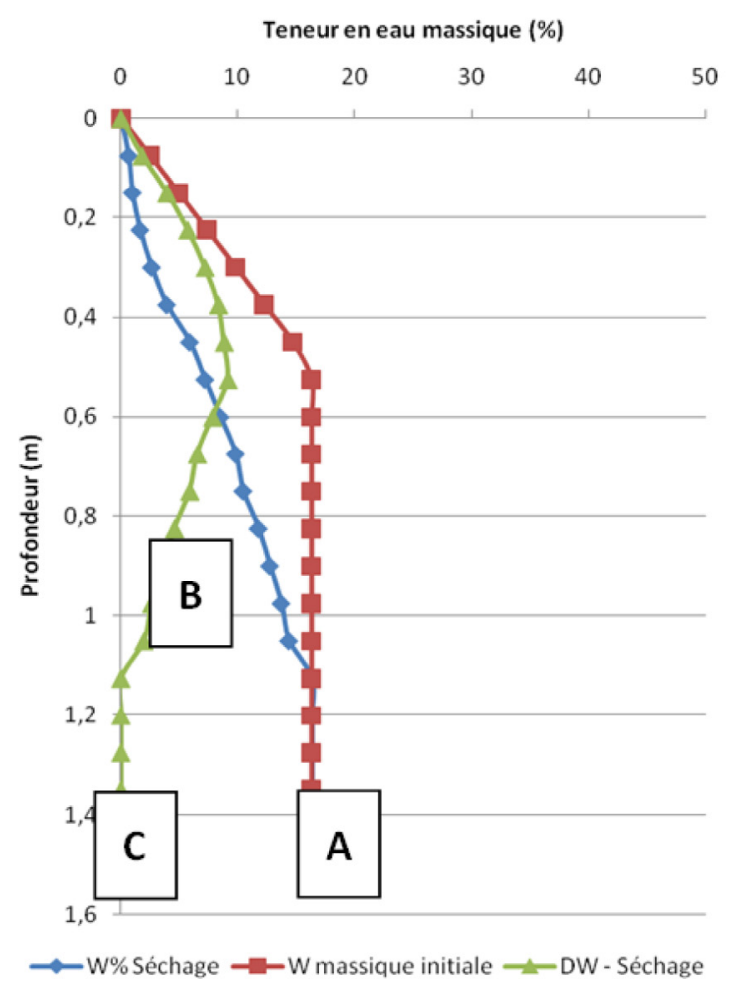

\section{Cycle "humidification"}

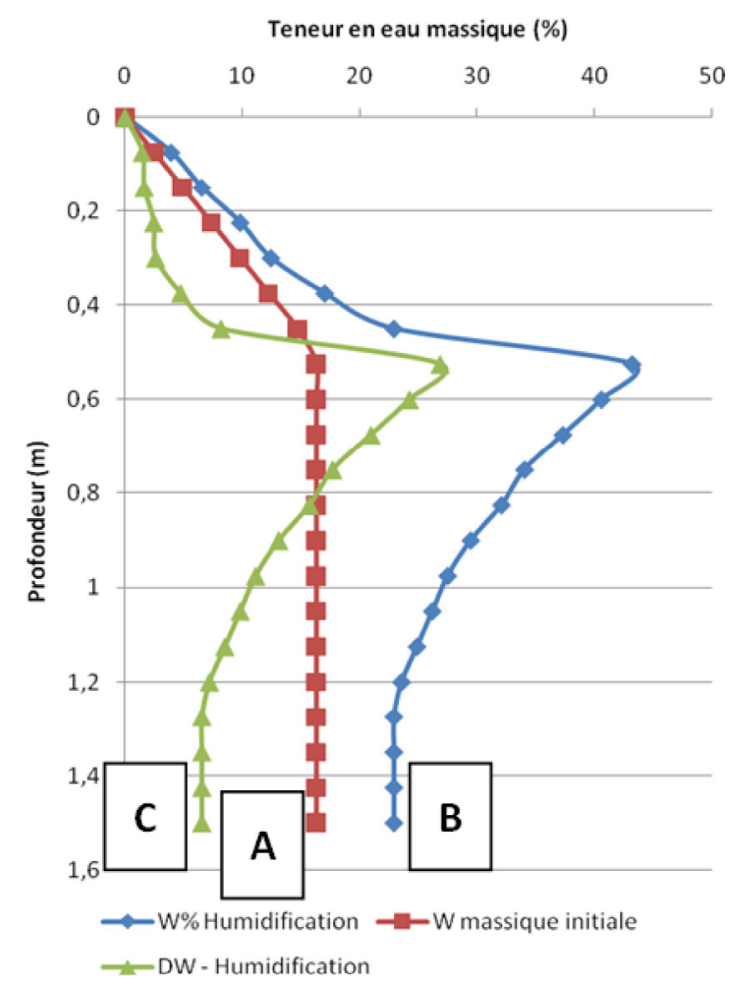

Fig. 9. Exemple de teneurs en eau initiale et après périodes sèche et humide pour le site de Pessac (mesure par sondes TDR ${ }^{\mathrm{f}}$ ).

Fig. 9. Example of three water content distribution curves (initial, dry and wet periods) for Pessac area.

les profondeurs de la couche étudiée. Toutefois, il convient de tenir compte de trois aspects :

- la variation de volume d'un sol argileux n'est pas uniquement une fonction de sa teneur en eau. En effet, elle est également conditionnée par l'état de contrainte dans le sol, par le sens du chemin (séchage ou humidification) et naturellement par le comportement rhéologique du sol. Dans le sens du séchage, le phénomène de diminution de volume du sol peut ainsi être accentué par la contrainte qui règne à la profondeur considérée. À l'inverse, dans le sens de l'humidification, la variation de volume pourrait se trouver réduite par la contrainte qui règne à cette même profondeur (Bigot et Zerhouni, 2000).

- la pression de gonflement que sera capable de développer le matériau réduira cependant fortement cette atténuation, jusqu'à l'annuler aux plus faibles profondeurs (Bigot et Zerhouni, 2000).la variation de volume n'est manifestement pas libre de toute contrainte mécanique externe dans le sol. Elle sera fortement réduite horizontalement ${ }^{\mathrm{d}}$ en comparaison du mouvement vertical ${ }^{\mathrm{e}}$. Une adaptation des informations issues de l'essai au MEBE est donc nécessaire.

\footnotetext{
$\overline{\mathrm{d}}$ Le phénomène de retrait et de gonflement pourrait ainsi conduire à une compaction horizontale du matériau et à une augmentation progressive de la valeur de $K_{0}$ (contrainte latérale/contrainte verticale), probablement limitée à 1 .

${ }^{e}$ Près de la surface, le mouvement d'une particule pourra ne pas être uniquement vertical si la variation d'humidité n'est pas homogène. Des composantes horizontales de mouvement et de force pourraient alors se développer.
}

Bien qu'il soit communément admis (Geremew et al., 2009), qu'à partir d'essais classiques de laboratoire, que les mécanismes de retrait et de gonflement sont en général très différents, notamment en terme de cinétique, nous considérerons que leurs amplitudes totales sont égales et opposées, pour une même variation d'humidité. C'est d'ailleurs ce que semblent montrer les mesures de mouvements de sol in situ réalisés dans le cadre du programme ANR-ARGIC (Vincent et al., 2009) sur le site de Mormoiron (Fig. 10 et 11).

Leroy et al. (2009) ont ainsi observé que le phénomène de retrait-gonflement était réversible comme le montre la Figure 11 qui représente l'évolution de l'épaisseur de la couche en fonction de sa teneur en eau massique à différentes périodes de l'année. On y observe que le retrait et le gonflement de l'argile du site de Mormoiron s'effectuent sur le même chemin, et ce pour différents cycles d'évolution de la teneur en eau.

\subsection{Relation entre la déformation volumique d'un échantillon et le tassement ou le gonflement d'un massif de sol}

Les images provenant du MEBE ne permettent pas d'avoir une visualisation tridimensionnelle des variations de volume puisqu'il n'est pas possible d'avoir une information directe

\footnotetext{
${ }^{\mathrm{f}}$ Le système produit une impulsion électromagnétique très courte appliquée au câble coaxial incluant la sonde TDR (time domain reflectometry) pour les mesures de l'eau dans le sol.
} 


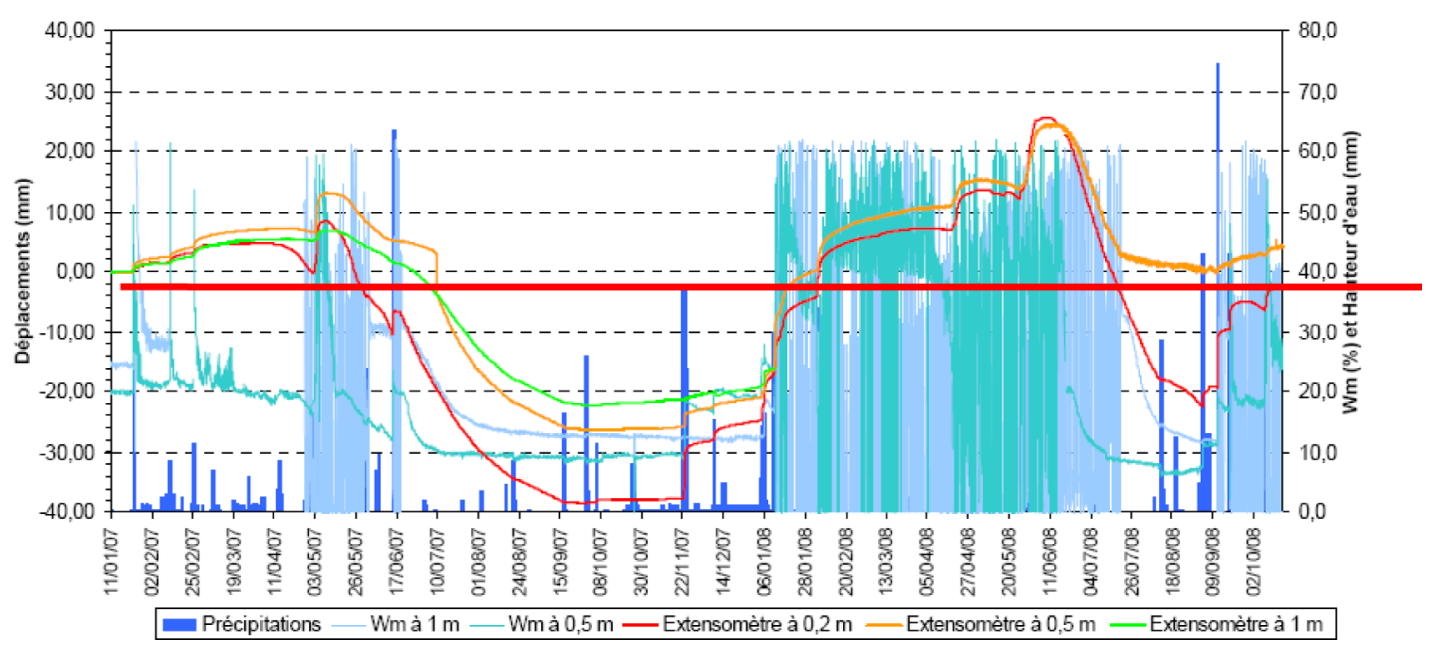

Fig. 10. Déplacements et teneurs en eau enregistrées à $0,2,0,5$ et $1 \mathrm{~m}$ de profondeur sur le site de Mormoiron.

Fig. 10. Displacements and water content recorded at 0.2-, 0.5- and 1-m depth, respectively, for Mormoiron site.

D’après Leroy et al. (2009).

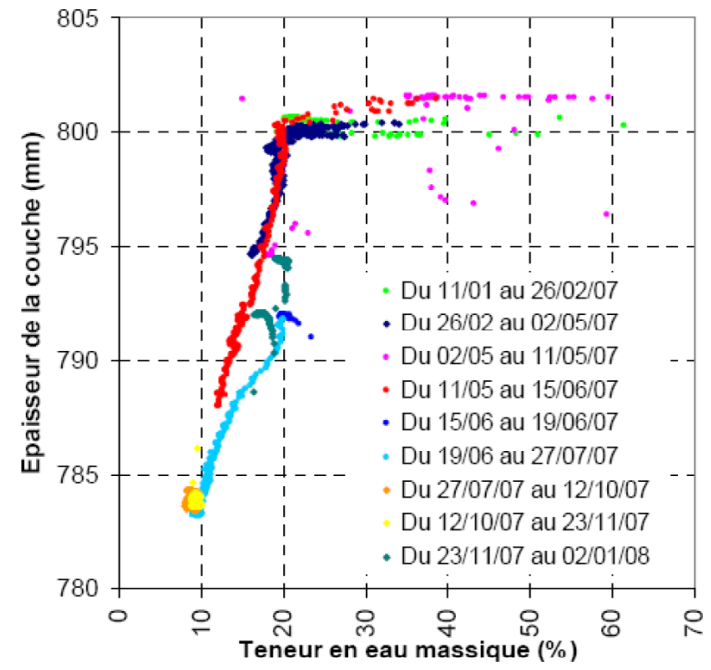

Fig. 11. Relation entre l'épaisseur de la couche de sol $0,2-1 \mathrm{~m}$ et la teneur en eau à $0,5 \mathrm{~m}$ sur le site de Mormoiron (valeurs à un pas de temps de $3 \mathrm{~h}$ ).

Fig. 11. Relation between the thickness of the soil layer and the water content at 0.5-m depth in the Mormoiron site.

D'après Leroy et al. (2009).

sur l'évolution de la topographie 3D de l'échantillon. Seule la variation de surface $\Delta S$ peut être observée. Il est donc nécessaire de faire une hypothèse, par exemple supposer une déformation isotrope, pour accéder à la déformation volumique totale de l'échantillon.

Dans le même ordre d'idée, mais cette fois au sein du massif, un volume élémentaire ne pourra pas se déformer de manière isotrope compte tenu de la présence de volumes élémentaires voisins, ce qui tend à limiter très fortement l'expansion horizontale du volume considéré.

C'est pourquoi la déformation verticale est prépondérante, en supposant que la pression de gonflement soit supérieure au poids des terres, ce qui est en général vrai à faible profondeur, là où les deux mécanismes (retrait et gonflement) s'expriment le plus.

Sur la base de ces considérations, et si l'on admet une déformation purement élastique et isotrope $(\varepsilon)$, l'observation de la déformation surfacique au microscope électronique vaut :

$$
\Delta S=2 \varepsilon \text { si } \Delta V=3 \varepsilon .
$$

Or, pour un cube de côté « $l »$ dont la déformation est bloquée horizontalement, la variation d'allongement $\Delta l$ dans la direction verticale vaut :

$$
\Delta l=(1+2 v) \times \varepsilon .
$$

Il existe donc un rapport :

$$
\mu=\frac{\Delta H}{\Delta \mathrm{S}}=\frac{(1+2 v)}{2}
$$

environ égal à 0,8 ( $v$ : coefficient de Poisson pris égal à 0,3 ; valeur classique pour une argile) entre le mouvement vertical attendu au sein du massif et la déformation surfacique observée au MEBE, dans les mêmes conditions de variation hydrique. Cette correction a été intégrée au modèle de comportement analytique.

\subsection{Proposition de profils hydriques}

Deux profils types (Fig. 13) de variation de la teneur en eau (séchage et hydratation) dans un massif de sol sont proposés, s'inspirant des mesures réalisées sur le site de Mormoiron (Fig. 12) dans le cadre du projet ARGIC.

Ces deux profils sont construits de la manière suivante :

- la valeur moyenne de teneur en eau du matériau sert d'axe de symétrie pour les courbes min (état sec) et max (état humide) - les courbes de variations de la teneur en eau sont des fonctions du type :

$$
w=\frac{a}{x}+b \quad x>0
$$

Pour la courbe correspondant à l'état sec (signe -$), w$ étant la valeur de la teneur en eau, $x$ la profondeur considérée, $a$ une 
Teneurs en eau massique min et max en fonction du temps sur le site de Mormoiron

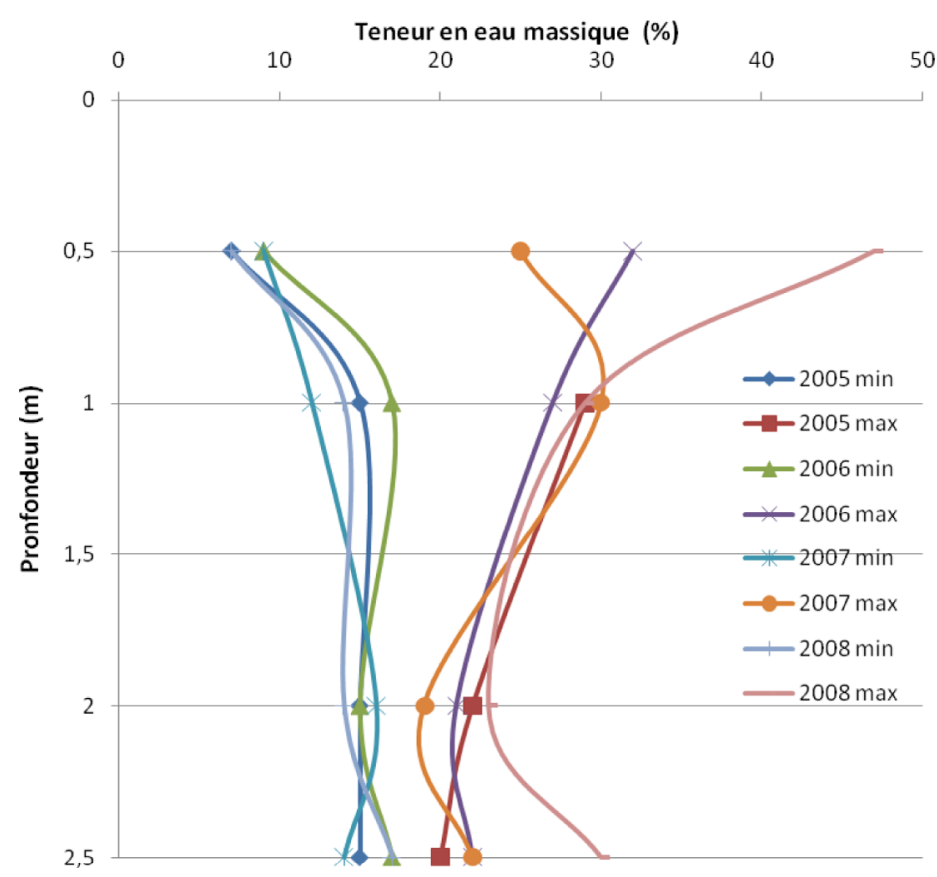

Fig. 12. Évolution de la teneur en eau en fonction du temps et de la profondeur sur le site de Mormoiron. Fig. 12. Water content evolution as a function of the depth at different times for Mormoiron site. Sur la base de données issues de Leroy et al. (2009).

\section{Profils "types" de teneur en eau ( $a=5$ et $b=20 \%$ )}

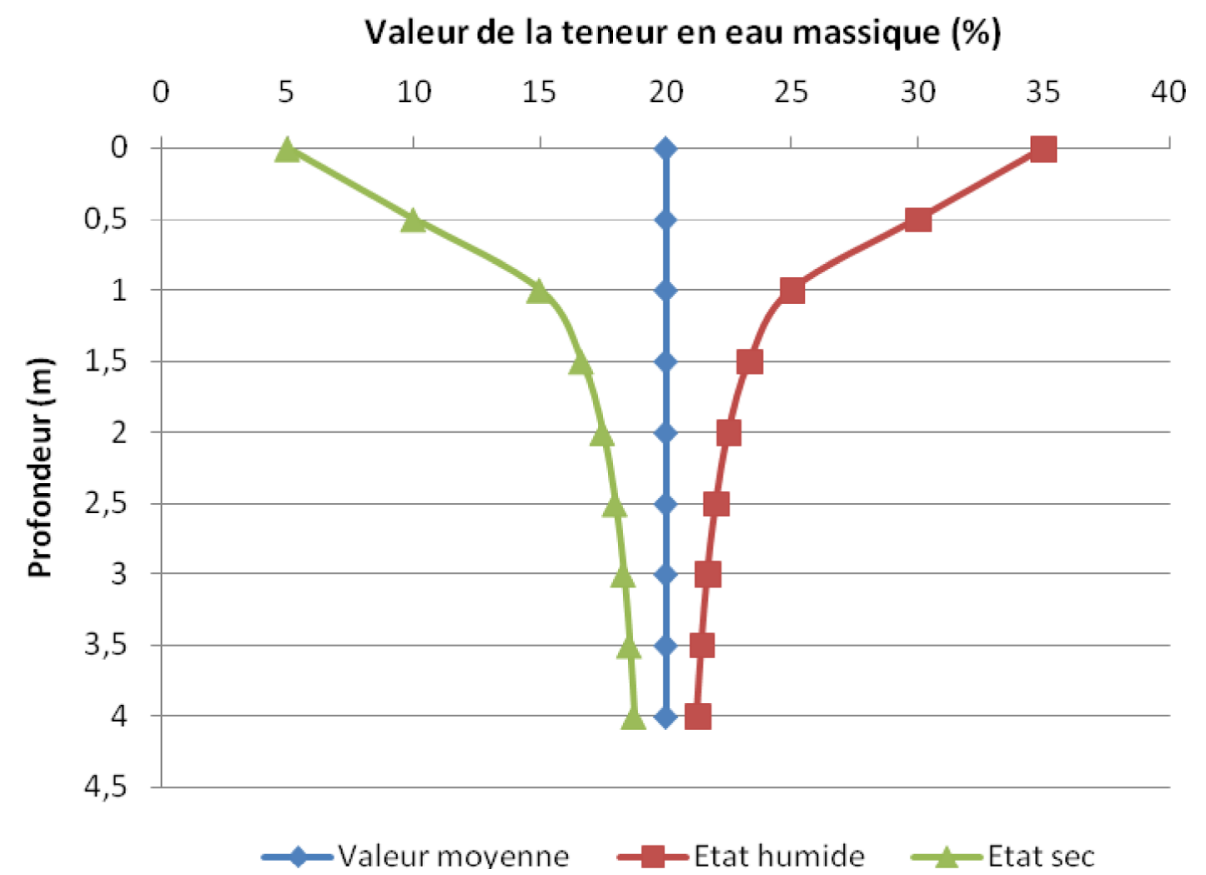

Fig. 13. Exemple de profils types de variation de teneur en eau (état sec et état humide) dans un massif de sol.

Fig. 13. Example of typical distributions of water content variation with depth (dry and wet states) into the soil. 


\section{Retrait et gonflement du massif de Montmorillonite Grecque}

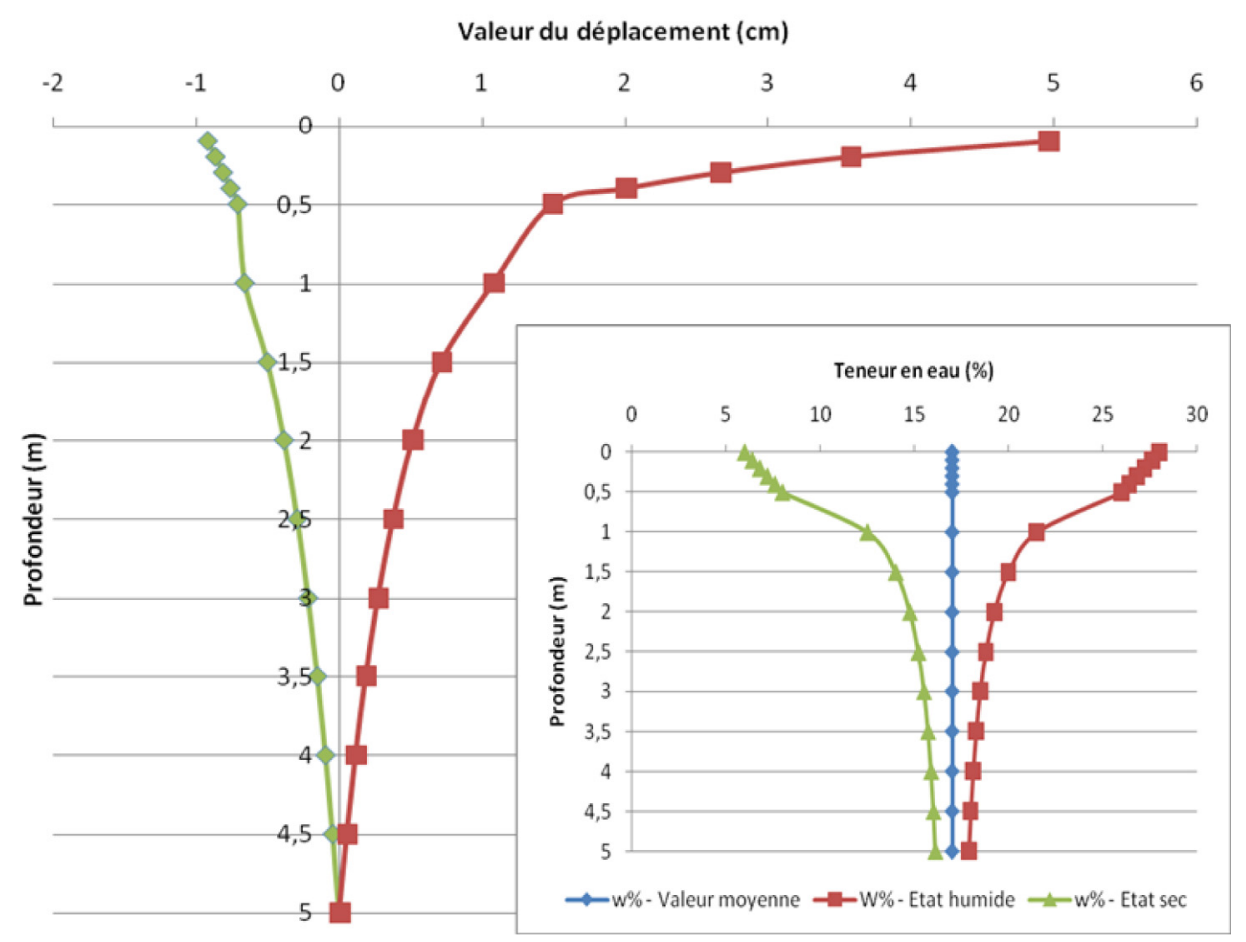

Fig. 14. Estimation du retrait et du gonflement de la couche de Montmorillonite grecque.

Fig. 14. Estimation of shrinkage and swelling of the Greek Montmorillonite layer.

constante à choisir et $b$ la teneur en eau moyenne. La valeur de la teneur eau en $x=0$, correspondant à la surface, sera définie pour assurer une « continuité » naturelle de la courbe. Pour la courbe correspondant à l'état humide (signe + ), les paramètres $a$ et $b$ sont identiques à ceux de l'état sec.

La Figure 13 illustre un exemple de ces deux courbes s'inspirant des observations du site de Mormoiron (Fig. 12). Pour ces exemples, les valeurs de $a$ et de $b$ sont respectivement de 5 et $20 \%$.

\subsection{Calculer à chaque profondeur le mouvement $\mathbf{H}_{\mathbf{i}}$ correspondant et le mouvement total $\mathrm{H}_{\max }$ en surface}

Des méthodes permettant de calculer l'amplitude du retrait et/ou du gonflement, en s'appuyant sur des essais de laboratoire existent. On peut citer par exemple les travaux de Philipponnat (1991) ou les préconisations de Bigot et Zerhouni (2000). L'approche présentée ici est différente puisqu'elle ne tient pas compte de l'état de contrainte.

La relation, qui relie la déformation surfacique du matériau à sa teneur en eau (Fig. 8), permet de calculer à chaque profondeur l'incrément de tassement ou de gonflement que subira l'épaisseur de couche élémentaire sous l'effet de la variation de teneur en eau.

On a ainsi pour chaque couche élémentaire :

- la valeur du tassement :

$$
\Delta H_{T i}=\mu \times\left[f\left(w_{i} \operatorname{moy}\right)-f\left(w_{i} \min \right)\right] \times H_{i} ;
$$

- la valeur du gonflement :

$$
\Delta H_{G i}=\mu \times\left[f\left(w_{i} \max \right)-f\left(w_{i} \operatorname{moy}\right)\right] \times H_{i} .
$$

Pour obtenir le tassement ou le gonflement de surface, il convient de sommer sur l'ensemble de la couche les incréments correspondants :

$$
H_{T_{\max }}=\sum \Delta H_{T i} \text { et } H_{G_{\max }}=\sum \Delta H_{G i} .
$$

Avec :

$-\mu:$ coefficient définissant la relation entre $\Delta H$ et $\Delta S$ (Sect. 4.3) ;

- $\mathrm{f}(w)$ : la relation reliant la teneur en eau à la déformation surfacique définie au sein du MEBE;

- $\Delta H_{T i}:$ l'incrément de tassement pour la couche élémentaire considérée de hauteur $H_{i}$;

- $\Delta H_{G i}$ : l'incrément de gonflement pour la couche élémentaire considérée de hauteur $H_{i}$;

$-H_{T_{\max }}$ : le tassement total en surface ;

$-H_{G_{\max }}$ : le gonflement total en surface.

Dans l'exemple présenté ici (Fig. 14), le couple de paramètres $a$ et $b$ utilisé vaut 4,5 et 17 respectivement pour la formule (5). Il permet de simuler une variation de teneur en eau massique en surface de 21 points (de 6 à $27 \%$ ) et une variation à $5 \mathrm{~m}$ de profondeur d'environ cinq points. On observe qu'audelà de $1 \mathrm{~m}$ de profondeur, la variation entre les profils « humide » et « sec » n'est déjà plus que de dix points environ. 
La Figure 14 présente également le résultat de calculs du tassement et du gonflement pour la Montmorillonite grecque. On constate que le potentiel de mouvement différentiel (gonflement-tassement) en surface est important $(6 \mathrm{~cm})$. Toutefois, à une profondeur d'environ $1 \mathrm{~m}$ à laquelle se situent généralement les fondations, cette valeur n'est plus que de $1,75 \mathrm{~cm}$. Il est à noter que, bien que les teneurs en eau des états « humide » et « sec » soient symétriques par rapport à une valeur moyenne de $17 \%$, la valeur du tassement calculée est très inférieure à celle du gonflement (dans un rapport de 5). $\mathrm{Ce}$ résultat est tout à fait cohérent avec la donnée expérimentale produite grâce au nouveau dispositif présenté dans cet article.

\section{Conclusions et perspectives}

L'innovation expérimentale présentée dans cet article constitue une avancée dans la caractérisation du comportement des matériaux sujets au retrait et au gonflement. Elle permet, à partir d'un échantillon de très petite taille, de définir la relation qui relie sa variation de déformation surfacique (ou volumique) à sa teneur en eau. Cet essai est très rapide en comparaison des essais classiques de laboratoire puisqu'il peut être réalisé en une journée environ (comparé à des essais de laboratoire classiques nécessitant parfois plusieurs semaines).

Nous avons montré que les résultats obtenus sur la Montmorillonite grecque sont compatibles avec ceux issus d'expérimentations réalisées en laboratoire par des essais classiques.

Un modèle analytique complète cet outil. Il propose une transition simple entre le comportement microscopique d'un échantillon argileux et le comportement du massif d'accueil, à l'échelle de l'ouvrage. Malgré les nombreuses hypothèses restrictives (représentativité de l'échantillon, réversibilité du retrait et du gonflement, effet d'échelle, indépendance de l'état de contrainte, etc.), le modèle analytique semble être en mesure de proposer des ordres de grandeur réalistes du retrait et du gonflement en surface et en profondeur.

Les perspectives d'évolution de l'innovation expérimentale concernent à la fois le MEBE lui-même et le système de pesée. En l'effet, l'utilisation d'un MEBE de haute résolution permettrait d'améliorer la qualité des images acquises à pression de vapeur d'eau élevée, et de faciliter par la même l'analyse d'images, voire peut-être de pouvoir l'automatiser.

Concernant le système de pesée proprement dit, l'axe de progrès majeur serait de le rendre moins « concepteurdépendant », donc plus facilement manipulable par des utilisateurs moins expérimentés, notamment via une amélioration de l'automatisation et de l'ergonomie générale.

Ces travaux, dont la validité doit être confirmée sur une palette plus large de sols argileux prélevés sur des sites affectés par le retrait-gonflement, ouvrent des perspectives intéressantes, que ce soit au niveau des essais de caractérisation d'usage dans la recherche et l'expertise ou au niveau de tests d'efficacité de produits inhibants sur sols gonflants.

\section{Références}

Alonso EE, Gens A, Josa A. 1990. A constitutive model for partially saturated soils. Géotechnique 40: 405-430.

Bigot G, Zerhouni MI. 2000. Retrait, gonflement et tassement des sols fins. Bull. Lab. Ponts Chaussées 4252: 105-114.

Cui YJ, Delage P. 1996. Yielding and plastic behaviour of an unsaturated compacted silt. Can. Geotech. J. 33: 291-311.

Fleureau JM, Soemitro R, Taibi S. 1992. Behavior of an expansive clay related to suction. In: Proc. 7th International Conference on Expansive Soils, Dallas, août, $n^{\circ} 1$, pp. 173-178.

Fredlund DG, Rahardjo H. 1993. Soil mechanics for unsaturated soils. New York: John Wiley and Sons, 517 p.

Geremew Z, Audiguier M, Cojean R. 2009. Analyse du comportement d'un sol argileux sous sollicitations hydriques cycliques. Bull. Eng. Geol. Environ. 68: 421-436, doi: 10.1007/s10064-009-0203-4

Kazmierczak JB, Maison T, Delalain P, Laouafa F. 2010. Brevet FR1054771 - 2010-06-16. Dispositif et procédé de caractérisation d'un matériau.

Leroy S, Plat E, Vincent M. 2009. Projet ARGIC (module B - tâches 11 à 14). Suivi expérimental in situ de l'évolution dans le temps des teneurs en eau et des déplacements verticaux dans les sols argileux sur deux sites. Rapport BRGM/RP-56990-FR, 72 p., 67 ill.

Maison T. 2011. Étude microscopique des phénomènes de retrait et de gonflement des argiles. Mémoire de thèse de doctorat, École centrale de Paris.

Maison T, Laouafa F, Fleureau JM. 2008. Analyse à l'échelle microet macroscopique des mécanismes de retrait et de gonflement des sols argileux. Laboratoire central des Ponts et Chaussées. In: SEC 2008: Symposium international sécheresse et constructions, 1-3 septembre 2008, Marne-la-Vallée. Paris : LCPC, pp. 155161.

Maison T, Laouafa F, Fleureau JM, Delalain P. 2009a. Analyse aux échelles micro- et macroscopique des mécanismes de dessiccation et de gonflement des sols argileux. In: Actes du $19^{e}$ Congrès français de mécanique, 24-28 août 2009, Marseille.

Maison T, Laouafa F, Fleureau JM, Delalain P. 2009b. Microscopic analysis of shrinkage and swelling mechanisms in clayey soils / Analyse microscopique des mécanismes de dessiccation et de gonflement des sols argileux. In: Hamza M, Shahien M, ElMossallamy Y, eds. In: Proceedings of the 17th International Conference on Soil Mechanics and Geotechnical Engineering, 5-9 October 2009, Alexandria, Egypt. Amsterdam: IOS Press, pp. 660-663.

Makki L, Duc M, Droniuc N, Reiffsteck P, Maloula A, Magnan JP. 2008. Essai de retrait pour une meilleure classification de la sensibilité des sols à la sécheresse. In: Sécheresse et construction SEC 2008, Symposium international, Paris, France.

Montes HG. 2002. Étude expérimentale de la sorption d'eau et du gonflement des argiles par microscopie à balayage environnementale (ESEM) et l'analyse digitale d'images. Strasbourg: École et observatoire des sciences de la terre.

Philipponnat G. 1991. Retrait-gonflement des argiles - Proposition de méthodologie. Rev. Fr. Géotech. 57: 5-22.

Plat E, Le Roy S, Vincent M, et al. 2009. Recommandation d'essais de laboratoire adaptés à la caractérisation de l'aptitude d'un sol argileux au retrait-gonflement. Annexe B18 au rapport de synthèse du projet ARGIC. Rapport BRGM/RP-57018-FR, 121 p., 40 ill., 4 ann.

Vincent M, Hédou F, Chirouze M, Plat E, Le Roy S. 2008. Cartographie à l'échelle départementale de l'aléa retrait-gonflement 
des sols argileux à des fins préventives. In: Sécheresse et construction SEC 2008, Symposium international, Paris, France, pp. 55-62.

Vincent M, Cojean R, Fleureau JM, et al. 2009. Rapport de synthèse final du projet ARGIC (Analyse du retrait/gonflement et de ses incidences sur les constructions). Projet ANR-05-PRGCU-005.
Rapport BRGM/RP-57011-FR en partenariat entre le Centre de géosciences, le BRGM, le LMSSMat, le CERMES, Fondasol, l'INERIS, le LAEGO, l'INRA, le LGCIE, le LCPC, Météo-France, le GHYMAC et l'université de Poitiers, 92 p., 29 ill., 6 tabl., 39 ann (sur CD Rom).

Citation de l'article : Jean-Bernard Kazmierczak, Tatiana Maison, Farid Laouafa, Patrice Delalain, Jean-Marie Fleureau. Un nouveau dispositif pour la caractérisation du retrait et du gonflement des sols argileux. Rev. Fr. Geotech. 2016, 147, 1. 